\title{
Potassium-intercalated single-wall carbon nanotube bundles: Archetypes for semiconductor/metal hybrid systems
}

\author{
C. Kramberger, ${ }^{1}$ H. Rauf, ${ }^{2}$ M. Knupfer, ${ }^{2}$ H. Shiozawa, ${ }^{3}$ D. Batchelor, ${ }^{4}$ A. Rubio,${ }^{5}$ H. Kataura, ${ }^{6}$ and T. Pichler ${ }^{1}$ \\ ${ }^{1}$ Faculty of Physics, University of Vienna, Strudlhofgasse 4, A-1090 Vienna, Austria \\ ${ }^{2}$ IFW Dresden, Helmholtzstraße 20, D-01069 Dresden, Germany \\ ${ }^{3}$ Advanced Technology Institute, University of Surrey, Guildford GU2 7XH, United Kingdom \\ ${ }^{4}$ BESSY, Albert-Einstein-Straße 15, D-12481 Berlin, Germany \\ ${ }^{5}$ Departamento de Física de Materiales, Facultad de Químicas, Centro Mixto CSIC-UPV/EHU, Universidad del País Vasco, \\ 20018 San Sebastián/Donostia, Spain and Donostia International Physics Center (DIPC), 20018 San Sebastián/Donostia, Spain \\ ${ }^{6}$ Nanotechnology Research Institute, AIST, Tsukuba 305-8562, Japan
}

(Received 17 March 2009; revised manuscript received 28 April 2009; published 29 May 2009)

\begin{abstract}
The electronic properties of matter are on the nanometer scale strongly influenced by its local environment. The site-dependent electronic properties in composites of semiconducting and metallic matter are at the heart of establishing molecular electronics. Our combined theoretical and experimental investigations comprise first-principles calculations, resonant $\mathrm{x}$-ray absorption as well as core-level and valence-band photoemission on pristine and potassium intercalated bundles of mixtures of semiconducting and metallic single-wall carbon nanotubes. We present a coherent picture of the archetypical changes in the site selective electronic properties of this tunable semiconductor/metal hybrid system.
\end{abstract}

DOI: 10.1103/PhysRevB.79.195442

PACS number(s): 73.20.Mf, 73.22.-f, 78.20.Bh

\section{INTRODUCTION}

Single-wall carbon nanotubes (SWNT) are unique onedimensional allotrope of $s p^{2}$ carbon. They are archetypical prime examples of one-dimensional solids. Their inherent one-dimensional electronic confinement has stimulated an ever growing interest in basic and applied science. Interestingly a SWNT may either be a metallic (mSWNT) or a semiconducting (scSWNT). A SWNT may be envisaged as a rolled up stripe of graphene. It is metallic if one of its parallel cutting lines that slice the two-dimensional Brillouin zone of graphene hits the $K$ point at the zone boundary. Otherwise it is a semiconductor. Bulk bundles of $\mathrm{m} / \mathrm{sc} \mathrm{SWNT}$ are therefore an archetypical example of a metal-semiconductor hybrid composite that allows to study the complex interplay of contact potentials, internal charge transfer, as well as local modifications of the work function (WF). All of these are for instance key issues for the conductivity in transparent electrodes. ${ }^{1}$ In addition to their intrinsic metalsemiconductor-ambivalency SWNT expose intriguing electronic properties, such as one-dimensional Van Hove singularities (VHS) (Refs. 2 and 3) in the electronic density of states (DOS) or the one-dimensional Tomanaga-Luttinger liquid (TLL) (Ref. 4) behavior. Experimentally VHS were evidenced in the differential conductance, ${ }^{5}$ by electronenergy-loss spectroscopy (EELS), ${ }^{6}$ by optical absorption spectroscopy (OAS), ${ }^{7}$ by valence-band (VB) photoemission spectroscopy (PES), ${ }^{8}$ and by resonant near-edge $\mathrm{x}$-ray absorption spectroscopy (XAS). ${ }^{9}$ The TLL and its characteristic power-law scaling have been confirmed by transport studies, ${ }^{10}$ VB PES, ${ }^{8}$ and nuclear magnetic resonance (NMR). ${ }^{11}$

Alkali-metal intercalation has been demonstrated to shift the Fermi level into the conduction band (CB) of graphite. ${ }^{12}$ In this way the $\mathrm{CB}$ becomes accessible to VB methods such as angle-resolved PES. ${ }^{13,14}$ Alkali-metal intercalated SWNT are a compound system with a faceted variety of tunable vibronic and electronic properties. ${ }^{15-20}$ The tunable doping level modifies crucial fundamental properties such as the allowed optical transitions, the phonon spectrum, the bulk conductance, the internal charge neutrality level, the power-law scaling of the TTL, and the collective free-electron plasma frequency. This list highlights the rich blend of physics in semiconductor/metal composites and points directly at the urge for a comprehensive description of the interactions in alkali-metal/SWNT compounds.

Here we report on in-depth spectroscopic studies of potassium intercalated SWNT. The applied and discussed methods comprise first-principles calculations, core level and VB PES, and resonant near-edge XAS as well as EELS. We identify several successive regimes in $n$-type doped SWNT. At low doping levels only the mSWNT are doped, which shifts the metallic versus the semiconducting WF in VB PES. At intermediate doping levels the semiconducting SWNT are driven metallic, which homogenizes the chemical potentials and is reflected in an increased Doniach-Sunjic asymmetry in core-level PES and accompanied by a decay of the TLL power-law renormalization in VB PES. After the Fermi levels are pinned at the rising edge of the first unoccupied semiconducting VHS $\left(\mathrm{S}_{1}^{*}\right)$ the first and second semiconducting VHS are gradually populated in the intermediate doping regime. This gradual population of the VHS is explicitly evidenced by the gradual depletion of the corresponding $\mathrm{C} 1 \mathrm{~s}$ core-level excitations in XAS. At high doping stages even the VHS of the metallic SWNT are populated and a traditional bulk free-electron gas is established. The latter is confirmed by the sudden emergence of a free-charge carrier plasmon in EELS.

\section{EXPERIMENTAL}

We used the same batch of SWNT material as used and described previously. ${ }^{8,19}$ The narrow $(\sim 0.1 \mathrm{~nm})$ Gaussian- 


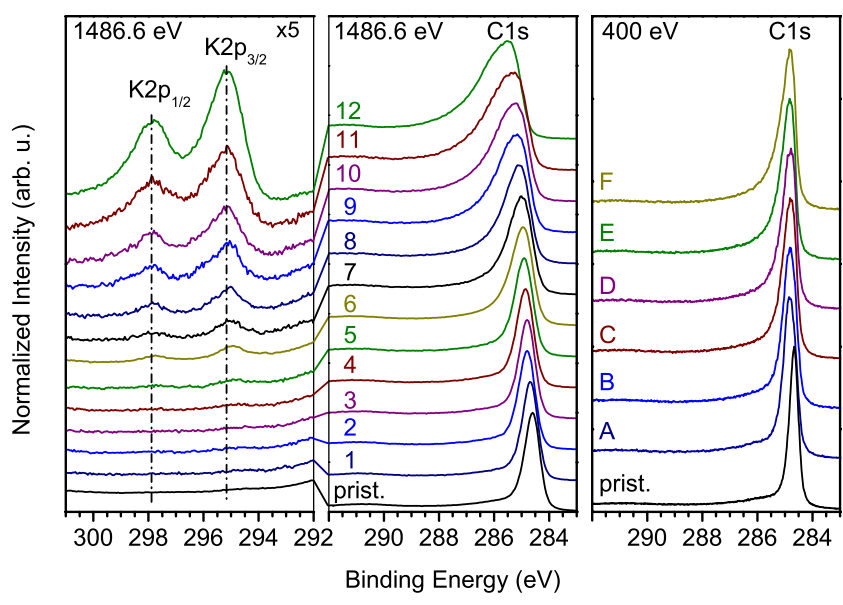

FIG. 1. (Color online) Photoemission spectra of the C $1 s$ and the split K $2 p$ core-level excitations as recorded at $\mathrm{Al} K \alpha=1486.6 \mathrm{eV}$ and the $\mathrm{C} 1 s$ line at $400 \mathrm{eV}$ photon energy of pristine (bottom) and successively potassium intercalated SWNT above.

diameter distribution is centered at $\sim 1.4 \mathrm{~nm}$. Intercalation series of drop-coated films of SWNT were performed in the laboratory facility at the IFW-Dresden and at beamline Ue 52 at BESSY II. The laboratory facility is equipped with an Al $K \alpha(1486.6 \mathrm{eV}) \mathrm{x}$-ray tube and a He gas lamp $(21.22 \mathrm{eV})$. Core-level PES and XAS were conducted with the tunable synchrotron light source at BESSY II. All photoemission experiments employed hemispherical Scienta SES 200 analyzers. XAS spectra were recorded via the drain current. The plane grating monochromator of Ue52 has a resolving power $^{21}$ of $E / \Delta E \sim 4 \times 10^{4}$. The VB PES spectra and Fermi edges were measured at low temperature $T=35^{\circ} \mathrm{K}$. Corelevel excitations were measured at ambient temperature since their resolution is not affected by temperature. ${ }^{9}$ We used commercial SAES getters to intercalate the samples in situ. During the potassium evaporation and for another $30 \mathrm{~min}$ the samples were held at $150{ }^{\circ} \mathrm{C}$ to equilibrate the potassium concentration in the surface layers. Freshly evaporated $\mathrm{Au}$ films were used to calibrate the work functions of the analyzers as well as the spectral intensity of the synchrotron source. For the calculation of the WF of pristine and doped tubes (isolated and bundles) we performed first-principles calculations based on density-functional theory (DFT) in the local-density approximation (LDA) (Calculations were done with the ABINIT code: http://www.abinit.org). The electronion interaction is described by norm conserving TrouillerMartins pseudopotentials and the wave functions are expanded in-plane waves with an energy cutoff at 40 Hartree and for the case of isolated tubes we used $20 k$ points to sample the BZ whereas for the bundles we used a Monkhorst-Pack grid of $3 \times 3 \times 12$.

\section{RESULTS AND DISCUSSION}

The core-level PES response of potassium intercalated SWNT is shown in Fig. 1. The C $1 s$ line shape undergoes significant changes and becomes more and more asymmetric as the intensity of the $\mathrm{K} 2 p$ peaks emerges. The peak areas at
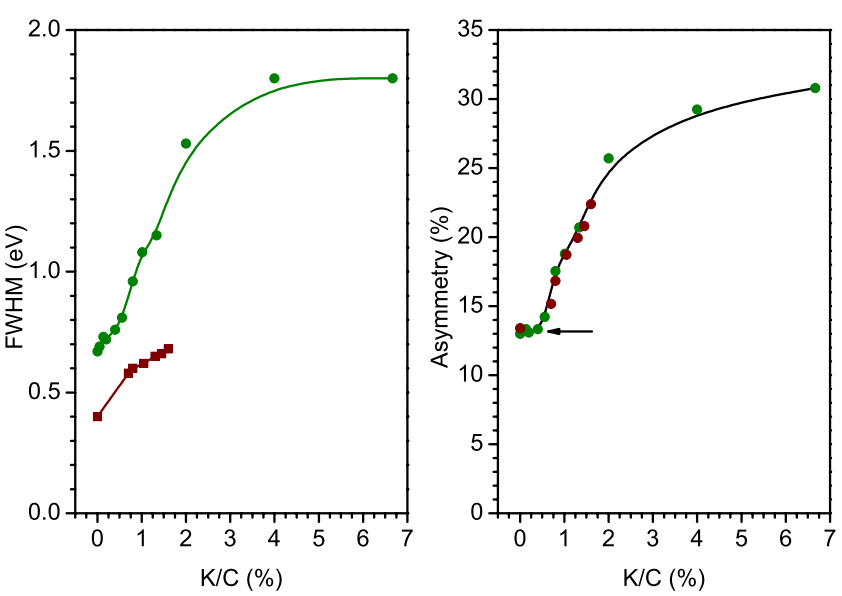

FIG. 2. (Color online) Linewidth (full width half maximum) and asymmetry $(\alpha)$ of the $\mathrm{C} 1 s$ core-level photoemission line at $1486.6 \mathrm{eV}$ (circles) and $400 \mathrm{eV}$ (squares). The asymmetries are calibrated for the Shirley background from the pristine samples. The arrow marks the onset of the intermediate doping regime.

Al $K \alpha(1486.6 \mathrm{eV})$ excitation have been used to determine the potassium concentration from the relative photoionization cross sections. At the excitation energy of $400 \mathrm{eV}$ the carbon core level has a much higher cross section as compared to the potassium core level, which complicates the direct assessment of the $\mathrm{K} / \mathrm{C}$ ratio. For a more quantitative comparison and access to the actual potassium concentrations at $400 \mathrm{eV}$ a line shape analysis with a single DoniachSunjic line was conducted. The line shape analysis provides reliable values for the line position, the linewidth, and most important the asymmetry $\alpha$ of the $\mathrm{C} 1 s$ line. The pristine material has been used as a reference for the Shirley background. The changes in $\alpha$ resemble the electronic DOS in the vicinity of the Fermi level ${ }^{22}$ and are hence the adequate quantity to compare independent in situ potassium intercalation series.

The linewidth and asymmetry of the $\mathrm{C} 1 s$ response from Fig. 1 are plotted as a function of doping level in Fig. 2. The potassium concentrations were determined from the direct comparison of the increase in the asymmetry. Within the rigid band shift model additional electrons just occupy the $\mathrm{CB}$ and shift the Fermi level, but the electronic band structure itself is retained. Following this scheme one can expect (at least) two adjacent doping regimes in a mixture of scSWNT and mSWNT. At small charge transfers the Fermi level does not yet touch the CB of the scSWNT and the add-electrons are exclusively put onto the mSWNT. But already at moderate charge transfers the Fermi level will also reach the $\mathrm{CB}$ of the scSWNT and will turn them metallic, too. As an immediate consequence one would expect a retained level of metallicity throughout the low doping regime. In fact the early doping steps below $\sim 5 \%$ o show much weaker changes in their asymmetry $\alpha$. The conservative $\alpha$ is concomitant to a gradually growing linewidth. There is a steep change in this overall behavior at $\mathrm{K} / \mathrm{C}$ ratios from $\sim 5 \%$ o to $\sim 10 \%$ o. Here after the linewidth, peak position, and the asymmetry are increasing significantly. This steep change is identified as the transition from the low doping regime to 

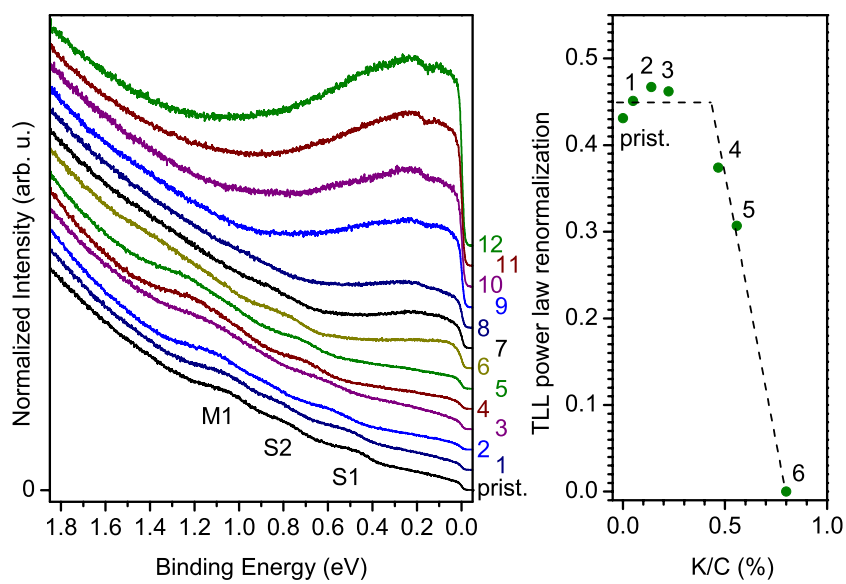

FIG. 3. (Color online) Left panel: VB photoemission spectra of intercalated SWNT as recorded at $21.22 \mathrm{eV}$ photon energy of pristine (bottom) and successively potassium intercalated SWNT (above). Right panel: power-law scaling parameter of the Tomanaga-Luttinger liquid vs doping level. The figure is reproduced from Ref. 19.

the intermediate doping regime. This transition correlates quantitatively to the crossover from the TTL to a Fermi liquid from $\sim 5 \%$ o to $\sim 10 \%$, where the decay of the TTL power-law renormalization was experimentally found in VB PES. ${ }^{19}$ The VB spectra of pristine and successively potassium doped SWNT are shown next to the decay of the power-law renormalization in Fig. 3. At low doping levels the diameter cumulative VHS $\left(\mathrm{S}_{1}, \mathrm{~S}_{2}, \mathrm{M}_{1}\right)$ in the VB of bulk SWNT are well resolved at $\sim 0.45 \mathrm{eV}, \sim 0.75 \mathrm{eV}$, and $\sim 1.05 \mathrm{eV}$ binding energy. In the low doping regime these cumulative VHS are retained but they are shifted. Furthermore, a closer look reveals that the shift in binding energies is stronger in semiconducting SWNT. To the end of the low doping regime the $S_{2}$ and the $M_{1}$ peaks have coalesced and the shifted $S_{1}$ peak just touches the pristine $S_{2}$ peak. The relative shifts of the scSWNT and the mSWNT correspond to a Fermi-level pinning just at the rising edge of the $S_{1}^{*}$ peak in the $\mathrm{CB}$. The dynamic rearrangement of the chemical potentials between semiconducting and metallic SWNT in the low doping regime is a clear experimental observation and lies naturally beyond a straightforward rigid band shift. Of course the rigid band shift is still valid for the individual SWNT, but the bulk compound system is more complex.

Both the asymmetry $\alpha$ of the $\mathrm{C} 1 s$ line as well as the power-law scaling of the $\mathrm{VB}$ directly relate to the actual number of metallic SWNT in the bundle. As the variety of scSWNT is gradually turned metallic the power-law scaling vanishes and the asymmetry increases steeply. In the following intermediate doping regime the metallicity and asymmetry continue to grow. The binding energies of VB VHS simply shift in parallel, which points at a restored rigid band shift. The homogeneous intermediate doping regime extends up to potassium concentrations of $\sim 20 \%$, where the VHS disappear and the electronic DOS at the Fermi level increases steeply. At this doping level the Fermi level is finally shifted into the VHS in the CB of the mSWNT. The applicability of the rigid band shift is only given prior to the high doping regime, where the band structure is apparently modi-

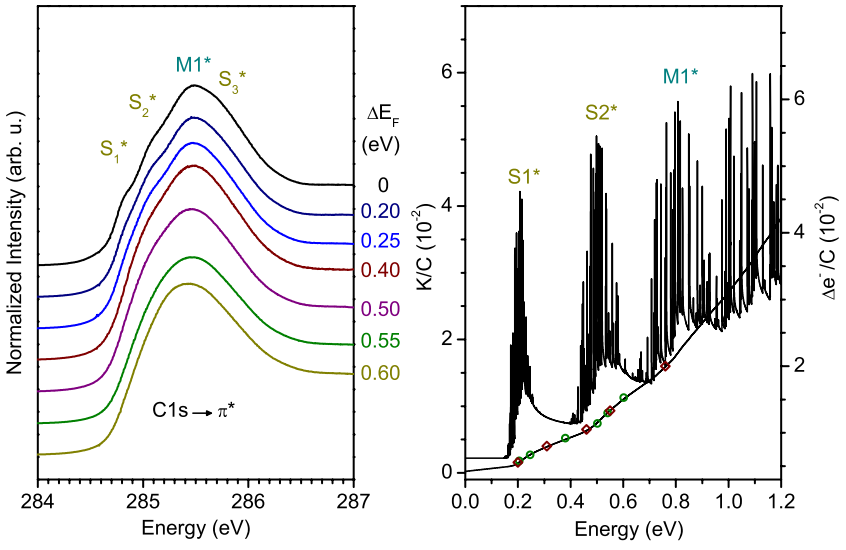

FIG. 4. (Color online) Left panel: x-ray response of the C 1 s $\rightarrow \pi^{\star}$ absorption edge of pristine (top) and successively potassium intercalated SWNT below. The fine structures are successively depleted with progressing doping steps. Right panel: diameter cumulative CB DOS of SWNT and Fermi-level shifts within the intermediate doping regime. Diamonds: PES experiments at $\mathrm{Al} K \alpha$ $1486.6 \mathrm{eV}$ excitation. Circles: PES at $400 \mathrm{eV}$ excitation and XAS.

fied as the characteristic VHS disappear and after the low doping regime, where intrabundle contact potentials rearrange the relative binding energies of scSWNT and $\mathrm{mSWNT}$.

The intermediate doping regime is thus the valid range of the rigid band shift and is just where the CB of scSWNT is gradually occupied. The steadily increasing diameter cumulative DOS of the CB of bulk SWNT is presented in the right panel of Fig. 4. The diameter cumulative DOS was derived by averaging the individual tight-binding DOS (Ref. 23) of all contributing chiralities. The cumulative peaks and individual spikes are located on top of a linear slope. The integrated area has been used to evaluate Fermi-level shifts from the doping levels that could be extracted from PES. The six individual shifts from $\sim 0.2$ to $0.6 \mathrm{eV}$ lie all in the intermediate doping regime and span the first two semiconducting VHS. Resonant XAS directly accesses the site selective transition from C $1 s$ core level into the SWNT CB. The spectra are in the left panel of Fig. 4. The depletion of these corelevel excitations is witnessed by the gradual depletion of the VHS in C $1 s$ absorption edge. The pristine absorption edge reveals the four SWNT specific fine structures ${ }^{9}$ of the $S_{1}^{*}, S_{2}^{*}$, $\mathrm{M}_{1}^{*}$, and $\mathrm{S}_{3}^{*} \mathrm{VHS}$ in the CB. The individual widths of the components increase with the doping levels and, more important, their relative intensities undergo sequential depletion. A closer look on the distinct changes in individual doping steps Fig. 4 reveals a detailed correspondence far beyond qualitative arguments. The pristine $\mathrm{C} 1 s$ edge shows the sequence of all four VHS peaks. The first doping step lies in the rising side of the $S_{1}^{\star}$ peak. Indeed, precisely this peak is noticeable reduced as compared to the pristine case. In the second step the $S_{1}^{\star}$ peak is already greatly suppressed and the corresponding Fermi-level shift $\sim 0.25 \mathrm{eV}$ is expected close to the falling edge of the peak. In the next step at $\sim 0.4 \mathrm{eV}$ the Fermi level lies in between the $S_{1}^{\star}$ and the $S_{2}^{\star}$ and the $S_{1}^{\star}$ peak is gone while the $S_{2}^{\star}$ peak is evidently preserved. In the next step the Fermi level is located in the rising edge of the $\mathrm{S}_{2}^{\star}$ VHS and the corresponding peak is diminished but still 


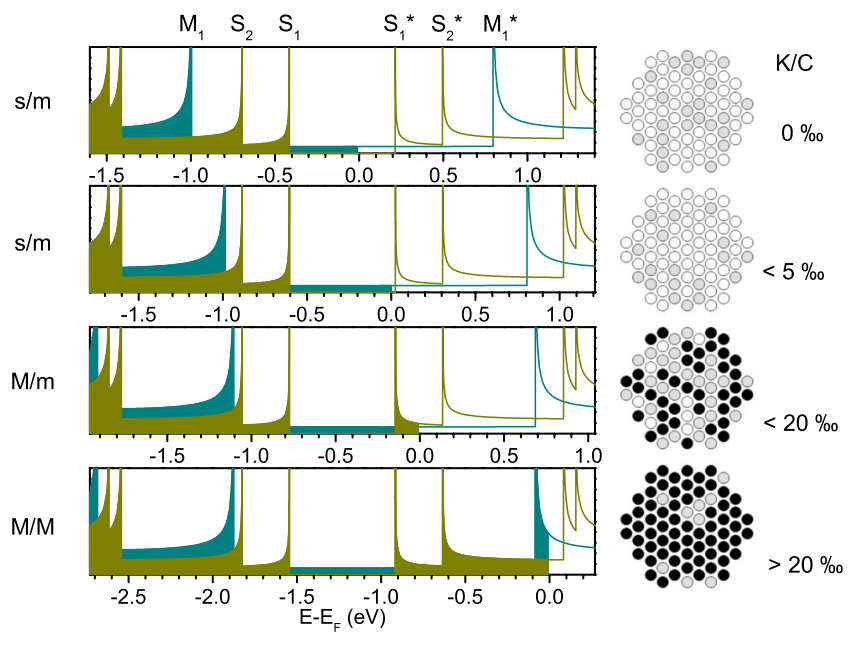

FIG. 5. (Color online) Right column: sketch of the electronic DOS in scSWNT and mSWNT, respectively. The individual stages are from top to bottom pristine, low doping, the transition to intermediate doping, intermediate doping, and high doping. Open circles represent semiconducting, gray circles normal metallic, and black circles doped SWNT with a high metallicity. Left column: sketches of the respective intrabundle contact potentials and their Fermilevel shifts.

present. In the second last step there is possible a faint and very broad reminiscence of $\mathrm{S}_{2}^{\star}$ left, which is definitely gone at $\sim 0.6 \mathrm{eV}$. As the doping level increases there is also an ongoing broadening of all components which would make a stand alone interpretation of the last two steps at least arguable, however in the present case they are a consequent continuation of the evident observation in the preceding doping steps.

While XAS can very well access the changes in the intermediate doping regime, we will turn on EELS to discuss the following high doping regime. There is another intriguing transition that was observed in an EELS study on potassium intercalated SWNT by Liu and co-workers. ${ }^{17}$ The Drude plasmon of free-charge carriers arises instantly between concentrations of $\mathrm{K} / \mathrm{C} \sim 40 \%$ and $\mathrm{K} / \mathrm{C} \sim 50 \%$ o. We suggest this transition marks the completion of the high doping regime, where all mSWNT are doped beyond their first VHS and are then in, our terminology, MSWNT. Unless a mSWNT is highly doped, it is just minority metal with a small DOS at the Fermi energy $\left(E_{F}\right)$. Doped scSWNT as well a sufficiently heavily doped mSWNT will be good metals and hence MSWNT. At the point where the vast majority of the SWNT has been turned to MSWNT the Fermi liquid of quasi-free electrons is no longer perforated and pinned by depleted channels of mSWNT.

The doping scenario of the archetypical metal/ semiconductor composite comprises three successive doping regimes and the distinction of scSWNT, normal mSWNT, and doped MSWNT. The conceptual idea is visualized in Fig. 5, where s/m denotes the pristine sc/mSWNT and M/M denotes highly doped SWNT with a high DOS at $E_{F}$.

In PES we observe a slightly less than $0.1 \mathrm{eV}$ higher work function for scSWNT. This observation is fully consistent to ours and others ${ }^{24-26}$ calculations of the diameter-dependent
WF in mSWMT and scSWNT. We looked at bundles of all metallic or semiconducting monochiral zigzag tubes doped with different alkali metals, as well as at alkali-metal doped dimers of metallic and semiconducting zigzag tubes. The latter are a model system to investigate how the tube-tube interactions fix the Fermi-level position with respect to the middle of the semiconducting gap. Here we find that in pristine bundles the Fermi level of the metallic tube moves slightly toward the bottom of the conduction band of the semiconducting tube. The diameter-dependent effect reproduces the experimentally observed $0.1 \mathrm{eV}$ for a mean diameter of $1.4 \mathrm{~nm}$. Regarding the doping dependence, the computed WF is either in monochiral or $\mathrm{sc} / \mathrm{m}$ dichiral bundles basically insensitive to the detailed configurations of the intercalated atoms, and merely a function of the intercalatant concentration. For high doping the WF and density of states at the Fermi level become indistinguishable for monochiral metallic and semiconducting tubes bundles.

In the low doping regime the charge transfer occurs exclusively onto the mSWNT, which in turn shifts the intrabundle contact potentials and causes the relative shifts of the VHS in VB PES. At the transition to the intermediate doping regime from $\sim 5 \%$ o to $\sim 10 \%$ o the asymmetry $\alpha$ of the $\mathrm{C} 1 s$ line goes up and the TTL power-law scaling vanishes as more and more scSWNT are turned into metals and the isolation of the mSWNT is quickly lost. In experiment we are naturally dealing with a narrow but not negligible spread of chiralities and diameters which smears out all transitions of the doping regimes. The gradual population of the $\mathrm{CB}$ is directly observed in XAS. The low metallicity in the mcSWNT is maintained up to $K$ levels of $\sim 20 \%$ o. In the following high doping regime the broad $\mathrm{M}_{1}^{*}$ of the mSWNT is gradually populated, too. The characteristic VHS from the original band structure of SWNT are lost, which points at increased mutual electronic interactions among the individual SWNT. Finally, at $\sim 50 \%$ o the majority of the mSWNT are doped MSWNT with an improved metallicity and the percolative transition to a normal three-dimensional Fermi liquid with a characteristic Drude plasmon is completed.

The above presented scenario of doping regimes in a bulk sample of SWNT is consistent to our first-principles calculations on mono- and dichiral-zigzag bundles and fully accounts for all the detailed and diverse spectroscopic signatures in core level and VB PES, XAS, and EELS. We point out that the heterogeneous electronic states in $\mathrm{m} / \mathrm{sc} / \mathrm{M}$ SWNT represent a well defined, yet sufficiently complex system to theoretically and experimentally address the electronic properties and interactions in bulk as well as molecular metal-semiconductor hybrid systems.

\section{SUMMARY}

We have presented and elucidated the detailed changes in the electronic properties of potassium intercalated SWNT and their respective spectroscopic signatures. The in-depth and comprehensive spectroscopic information allows to draw one conclusive picture of successive doping regimes and different types of metallicity in potassium intercalated SWNT. 
Already in the pristine composite of $d \sim 1.4 \mathrm{~nm} \mathrm{mSWNT}$ and scSWNT the Fermi energy is $\sim 0.1 \mathrm{eV}$ above the middle of the semiconducting gap. In the low doping regime below $\mathrm{K} / \mathrm{C} \sim 5 \%$ the WF of scSWNT and mSWNT are continuously rearranged due to the targeted charge transfer onto the mSWNT. The pristine offsets in binding energies as well as the following rearrangements are directly seen in valenceband PES. In the intermediate doping regime above $\mathrm{K} / \mathrm{C}$ $\sim 10 \%$ the intrabundle contact potential are canceled and the additional metallic states in doped SWNT are reflected in the asymmetry of the C $1 s$ core-level PES peak. The successive occupation of the VHS in the $\mathrm{CB}$ is experimentally verified in detail by high resolution XAS. In the high doping regime beyond $\mathrm{K} / \mathrm{C} \sim 20 \%$ the metallicity of the pioneering $\mathrm{mSWNT}$ is boosted as the Fermi level passes their first VHS. At $\mathrm{K} / \mathrm{C} \sim 50 \%$ o the metallicity of all different $\mathrm{mSWNT}$ is increased and a regular homogeneous Fermi liquid is established.

Gradual potassium intercalation opens a way to tickle the delicate balance of internal charge transfer and the local WF. The peculiar metallic phase that governs in the intermediate doping regime is ascribed to a nanoperforated Fermi liquid. While it exhibits the typical Fermi edge the collective freecharge carrier plasmon is completely suppressed by metallic minority channels. The Drude response only occurs after the minority mSWNT are turned into regular metals, too. Potassium-intercalated SWNT are a simple enough model system to render first-principles calculations feasible, but yet complex enough to clearly display the whole spectrum of Fermi-level pinning, site selective charge transfer as well as perforated and conventional bulk Fermi liquids. It allows a quantitative access to these fundamental phenomena of utmost technological relevance for urging applications such as transparent electrodes.

\section{ACKNOWLEDGMENTS}

We acknowledge technical support by R. Schönfelder, R. Hübel, and S. Leger. T.P. acknowledges DFG Grant No. PI 440 4. A.R. acknowledges funding by the Spanish MEC (Contract No. FIS2007-65702-C02-01), "Grupos Consolidados UPV/EHU del Gobierno Vasco" (Grant No. IT-319-07), and the EC through E-I3 ETSF project (Contract No. 211956) and NANO-ERA CHEMISTRY. We acknowledge support by the Barcelona Supercomputing Center, "Red Espanola de Supercomputacion" and SGIker ARINA (UPV/ EHU).
${ }^{1}$ M. W. Rowell, M. A. Topinka, M. D. McGehee, H. J. Prall, G. Dennler, N. S. Sariciftci, L. B. Hu, and G. Gruner, Appl. Phys. Lett. 88, 233506 (2006).

${ }^{2}$ N. Hamada, S.-i. Sawada, and A. Oshiyama, Phys. Rev. Lett. 68 , 1579 (1992).

${ }^{3}$ J. W. Mintmire, B. I. Dunlap, and C. T. White, Phys. Rev. Lett. 68, 631 (1992).

${ }^{4}$ M. Bockrath, D. H. Cobden, J. Lu, A. G. Rinzler, R. E. Smalley, L. Balents, and P. L. McEuen, Nature (London) 397, 598 (1999).

${ }^{5}$ T. W. Odom, J.-L. Huang, P. Kim, and C. M. Lieber, Nature (London) 391, 62 (1998).

${ }^{6}$ T. Pichler, M. Knupfer, M. S. Golden, J. Fink, A. Rinzler, and R. E. Smalley, Phys. Rev. Lett. 80, 4729 (1998).

${ }^{7}$ H. Kataura, Y. Kumazawa, Y. Maniwa, I. Umezu, S. Suzuki, Y. Ohtsuka, and Y. Achiba, Synth. Met. 103, 2555 (1999).

${ }^{8}$ H. Ishii, H. Kataura, H. Shiozawa, H. Yoshioka, H. Otsubo, Y. Takayama, T. Miyahara, S. Suzuki, Y. Achiba, M. Nakatake, T. Narimura, M. Higashiguchi, K. Shimada, H. Namatame, and M. Taniguchi, Nature (London) 426, 540 (2003).

${ }^{9}$ C. Kramberger, H. Rauf, H. Shiozawa, M. Knupfer, B. Büchner, T. Pichler, D. Batchelor, and H. Kataura, Phys. Rev. B 75, 235437 (2007).

${ }^{10}$ B. Gao, A. Komnik, R. Egger, D. C. Glattli, and A. Bachtold, Phys. Rev. Lett. 92, 216804 (2004).

${ }^{11}$ B. Dora, M. Gulacsi, F. Simon, and H. Kuzmany, Phys. Rev. Lett. 99, 166402 (2007).

${ }^{12}$ E. J. Mele and J. J. Ritsko, Phys. Rev. Lett. 43, 68 (1979).
${ }^{13}$ A. Gruneis, C. Attaccalite, L. Wirtz, H. Shiozawa, R. Saito, T. Pichler, and A. Rubio, Phys. Rev. B 78, 205425 (2008).

${ }^{14}$ A. Grüneis, C. Attaccalite, A. Rubio, D. V. Vyalikh, S. L. Molodtsov, J. Fink, R. Follath, W. Eberhardt, B. Büchner, and T. Pichler, Phys. Rev. B 79, 205106 (2009).

${ }^{15}$ A. M. Rao, P. C. Eklund, S. Bandow, A. Thess, and R. E. Smalley, Nature (London) 388, 257 (1997).

${ }^{16}$ A. Kukovecz, T. Pichler, R. Pfeiffer, and H. Kuzmany, Chem. Commun. (Cambridge) 2002, 1730.

${ }^{17}$ X. Liu, T. Pichler, M. Knupfer, and J. Fink, Phys. Rev. B 67, 125403 (2003).

${ }^{18}$ T. Pichler, X. Liu, M. Knupfer, and J. Fink, New J. Phys. 5, 156 (2003).

${ }^{19}$ H. Rauf, T. Pichler, M. Knupfer, J. Fink, and H. Kataura, Phys. Rev. Lett. 93, 096805 (2004).

${ }^{20}$ H. Shiozawa, T. Pichler, C. Kramberger, M. Rummeli, D. Batchelor, Z. Liu, K. Suenaga, H. Kataura, and S. R. P. Silva, Phys. Rev. Lett. 102, 046804 (2009).

${ }^{21}$ A. Scholl, Y. Zou, T. Schmidt, R. Fink, and E. Umbach, J. Electron Spectrosc. Relat. Phenom. 129, 1 (2003).

${ }^{22}$ S. Doniach and M. Sunjic, J. Phys. C 3, 285 (1970).

${ }^{23}$ H. Shiozawa, H. Ishii, H. Kataura, H. Yoshioka, H. Kihara, Y. Takayama, T. Miyahara, S. Suzuki, Y. Achiba, T. Kodama et al., Physica B 351, 259 (2004).

${ }^{24}$ J. Zhao, J. Han, and J. P. Lu, Phys. Rev. B 65, 193401 (2002).

${ }^{25}$ B. Shan and K. Cho, Phys. Rev. B 73, 081401(R) (2006).

${ }^{26}$ W. S. Su, T. C. Leung, and C. T. Chan, Phys. Rev. B 76, 235413 (2007). 